\title{
Effects of Point of Aim on the Accuracy and Eye Movement Behavior in Bowling: A Pilot Study
}

\author{
Jongil Lim', Seung Ho Chang ${ }^{2 *}$, Adriane Cris Tomimbang ${ }^{2}$ \\ ${ }^{1}$ Department of Counseling, Health and Kinesiology, Texas A\&M University - San Antonio, One University Way, San Antonio, TX, USA \\ ${ }_{2}^{2}$ Department of Kinesiology, San Jose State University, 1 Washington Square, San Jose, CA, USA
}

Corresponding Author: Seung Ho Chang, E-mail: seungho.chang@sjsu.edu

\section{ARTICLE INFO}

\section{Article history}

Received: April 12, 2018

Accepted: July 16, 2018

Published: July 31, 2018

Volume: 6 Issue: 3

Conflicts of interest: None

Funding: None

\begin{abstract}
Background: A common research question in far-target aiming has been the importance and significance of the final visual fixation before movement initiation. In rolling tasks, such as 10pin bowling, location of point of aim needs not be at the final target, the pins, but may be located at any point along the trajectory of the ball. Objective: Specific interest in the present experiment has focused on the relationship between visual point of aim and performance accuracy, and the relationship of visual control strategies utilized by expert performers. Methods: Skilled bowlers $(\mathrm{N}=7)$ performed 20 trials per condition concentrating on visual targets in different distances along the bowling lane (20, 40, 60 feet, and self-selected). Ball trajectory was tracked using a video based system and eye movement was measured using an eye tracking system. Results: Deviation of the ball from the visual target increased with visual target distance, while deviation of the ball from the pins was the lowest in the self-selected visual targets, followed by aiming at the pins. The final fixation duration before movement initiation was not associated with ball accuracy regardless of visual target locations. However, results demonstrated the association between final fixation duration task difficulty, that is, longer final fixation duration with increased visual target distance. Conclusion: The results indicate that visual fixations before movement initiation are uncharacteristically long while visual fixations just before the completion of the movement are relatively short.
\end{abstract}

Key words: Motor Skill, Attention, Accuracy, Vision, Fixation

\section{INTRODUCTION}

Bowling is a sport that competes the spatial accuracy of a projectile object, in order to aim a bowling ball correctly towards an intended target. The objective of the sport is to knock down as many pins as possible. The public generally enjoys ten-pin bowling, as a recreational league sport or an entertaining weekend activity, but there are competitive bowlers that train themselves to attain accurate and consistent bowling form (Razman, Abas, Osman, \& Cheong, 2011; Young, Sherk, \& Bemben, 2011). The projectile motion of a ball is typically divided into 5 sequences: targeting, push-off, down swing, back swing, and ball release, while the whole body is in forward propulsion parallel to the floor. Throughout the projectile motion of the ball, bowlers are instructed to focus on a target (or mark) to which they will project the ball (Taylor, 1979). A common strategy known as spot bowling is a technique in which the target arrows, embedded in the lane about 15 feet down from the foul line, are typically used as locations for aiming. Utilizing these seven arrows as a spatial reference increases the chance of consistently hitting the target pin and achieving the desired outcome. Studies examining the influence of visual behavior in far-target aiming consistently show that accurate performance is dependent on how vision and focus of visual attention is regulated in order to acquire task-relevant visual information (Vickers, 2011).

The pick-up of essential visual parameters during response preparation was particularly important and characterized by the final visual fixation before movement initiation. An earlier onset of the final eye fixation and longer final eye fixation duration are characteristics distinguishing skill differences in higher versus lower skilled performers and accurate versus inaccurate trial performance. However, in previous studies the location of visual focus and the target location were always coincident (e.g., basketball freethrow, golf putting, billiards, archery, and dart). In a rolling task such as 10-pin bowling, the point of aim need not be at the final target, the pins, but may be located at any point along the trajectory of the ball (Vickers, 2007). The advantages of paying attention on an external source of information have been demonstrated in many motor behavior studies (Wulf, 2007). In a variety of motor skills, age groups, and skill levels, focusing on the external cues such as intended movement effect showed benefits in performance outcome and learning than attending to internal cues such as one's 
body movements. Tasks demonstrating these effects include balancing (Wulf, McNevin, \& Shea, 2001), vertical jumping (Wulf, Dufek, Lozano, \& Pettigrew, 2010), golf chipping shot (Wulf \& Su, 2007), and dart throwing (Lohse, Sherwood, \& Healy, 2010; Marchant, Clough, \& Crawshaw, 2007). Advantages of externally focused attention were addressed in regards to reducing consciousness in controlling the movement and enhancing the smooth execution of movements (Wulf \& Lewthwaite, 2010). A few studies replicated the effectiveness of an external focus and further demonstrated that the performance and learning of motor skills was improved when attention was located to the external source of information further away from the body (Bell \& Hardy, 2009; Porter, Anton, Wikoff, \& Ostrowski, 2013). Particularly, for studies in which the far-target aiming task was employed (e.g., golf pitching shot; dart throwing), the group with a distal external cues (flight of the golf ball and bull's eye, respectively) displayed better accuracy compared to the internal cue group (own body movements) (Bell \& Hardy, 2009; McKay \& Wulf, 2012). However, in both studies participants were novice performers and one of the instructional cues used to focus the attention was difficult to visually focus on. That is, the imaginary attention was emphasized in external focus group (e.g., flight of the golf ball and flight of the dart, respectively).

While the beneficial effects of directing attention externally at a greater distance from the body have been demonstrated, studies that directly controlled the distance of visual focus of attention (e.g., focusing on the ball versus focusing on the hole throughout the entire stroke in golf putting) consistently showed no significant effect on distal attention of focus in performance (Cockerill, 1979) and learning (Mackenzie, Foley, \& Adamczyk, 2011). Moreover, after practicing the movements, the performance variability such as face angle, stroke path, and impact spot became equivalent between the two visual attention groups. Putter speed variability, indicating the golfer's ability to correctly estimate the required impulse, however, was further reduced in the distal focus group. This reduction in putter speed variability suggests the benefits of practicing, while visually focusing on the hole, can be amplified (Mackenzie et al., 2011). However, as authors discussed, different head positions might be associated with different levels of anxiety, more specifically "looking up" too soon during the stroke, particularly, when focus is on the ball. A stream of research in golf putting consistently demonstrated that use of characterized gaze control on the ball is an important strategy (Vickers, 1992, 2007, 2011; Vine, Moore, \& Wilson, 2011), which facilitates the brain to process task relevant attention for precise ball-club contact (Vickers, 2011). The intermediate spatial location between the performer and the target is frequently used as the location of aim. This location of aim is visually focused on if a projected object is required to travel along a curved path (e.g. breaking putts in golf and delivery of the curling stone), a technique also practiced in bowling. However, no studies have examined the effect of the distance of visual focus of attention, including intermediate targets, on the performance of far-target aiming. The purpose of this study, therefore, was to examine the robustness of the distance effect on visual focus of attention, more specifically through the direct manipulation of visual focus of attention in distance. Of particular interest in the present experiment were the relationship between the visual point of aim and performance accuracy, and the visual control strategies used by expert performers.

\section{METHOD}

\section{Participants and Design of Study}

Seven right-handed individuals volunteered for the study. Mean age was $21.14 \pm 1.06$ years and 3 participants were female and 4 were male. All subjects were skilled bowlers (average score $194.3 \pm 17.5$ ), as indicated by national collegiate competitive experience and also by Thomas, Schlinker, and Over (1996) (current average of 170 pins of more). Participants had normal or corrected-to-normal vision, were healthy, without any known oculomotor abnormalities, and signed an Institutional Review Board approved consent form. A descriptive research design (field observation) was utilized to examine the effect of visual target distance on spatial accuracy and fixation duration.

\section{Apparatus}

A standard 60-foot long, 40-inch wide, oiled synthetic lane was used for testing. Participants were allowed to use their own shoes and bowling balls. The bowling balls used in the study were regulated to be 8.59 inches in diameter, with a mass between 6 to 16 pounds. Two video cameras (Sony, Japan, Model DCR-TRV520) were positioned in the middle of the lane, facing down, to track ball trajectory from the transverse plane (Figure 1). One camera was directed at the pins (final target) the entire time and the second camera was focused at one of the intermediate targets. The position of the second camera was adjusted according to the order of intermediate target sequence. A third camera synced with the eye tracking system was positioned at the right side of the participants, in order to record the projectile motion of the ball from the sagittal plane (side-view).

Eye tracker (Applied Science Laboratories Series 5000 Head Mounted Eye Tracker) mounted on a helmet worn by the participant was used to record gaze movements. The line of gaze measured by a pupil and corneal reflection system was superimposed on the scene image recorded by an external scene camera. The point of gaze from the participant's view was recorded by the video recorder (JVC, Japan, Model SR-VS30). The sampling frequency was set at $60 \mathrm{~Hz}$, and the gaze position data was exported to the manufacturer provided software (ASL Eye-Trac 6000) for analysis.

\section{Procedures}

Participants were given six practice shots prior to the testing session. After completing the practice, the eye-tracking system helmet was put on the participant. Calibration was performed using a nine-point grid on the board placed directly in front of the participant. The participant took a four- or five-step approach and threw the ball underhand at the head pin (Figure 1). Participants were instructed to attempt to 
get the ball to hit the pins between the $17^{\text {th }}$ and $18^{\text {th }}$ boards, which is considered to be the optimal placement for bowling strikes (Taylor, 1979). Four distances of visual focus of attention were tested using a visual target at 20-, 40-, and 60 -feet down the lane, in addition to the participant's preferred location. The importance of examining these locations in the target process has been addressed; one at the end of the lane (pins), one at the end of the oil pattern (typically 40 feet down the lane), and one 15 to 25 feet down the lane (Slowinski, 2007). Participants chose the lateral position of their target for each condition (i.e. which board to aim for). Twenty trials were performed in each condition and three minutes rest between each condition was given. The order of condition was counterbalanced across participants. During each trial, the experimenter monitored each participant's gaze location and determined if location was indeed at the designated visual target location throughout ball projection.

\section{Data Analysis}

Temporal components of the bowling swing. For the sake of analyzing the fixation characteristics with respect to the movement execution phases (Figure 2), movement initiation time and release time were analyzed from images taken by the side-view camera (sagittal plane).

\section{Performance}

Spatial errors at the visual target and at the end of the lane were measured using frame-by-frame video analysis. Constant error, the signed horizontal deviation of the ball center relative to the visual targets $(20-, 40-$, and self-selected location) and relative to the pins (60 feet down the lane), was measured in inch of board. Absolute error and variable error were then computed.

\section{Eye movement}

A fixation is defined as an eye movement of less than $1^{\circ}$ of visual angle for $100 \mathrm{~ms}$ or longer. The average fixation duration and final fixation duration per trial were calculated. Fixations, which occurred between the push-off and ball release phases, were of specific interest. The last fixation in the preparation phase (e.g., before push-off) and the last fixation in the execution phase (e.g., before ball release) were analyzed and compared to average fixation.

\section{Statistical Analysis}

The effect of visual target distance on spatial accuracy and fixation duration was analyzed using one-way ANOVA with repeated measures (SPSS 16.0, Chicago, IL, USA). Data distributions were checked for normality and homogeneity of variances using the Shapiro-Wilk test and the Mauchly's test, respectively. In the case of violations of the sphericity assumption, $\mathrm{F}$ values were adjusted with the Greenhouse-Geisser procedures. A post-hoc analysis of the Bonferroni test was used to detect significant pairwise differences of means among visual target distances. A paired t-test (two-tailed) was used to determine if differences existed in absolute and variable error at the visual and final target in each visual target distance. Final fixation durations were

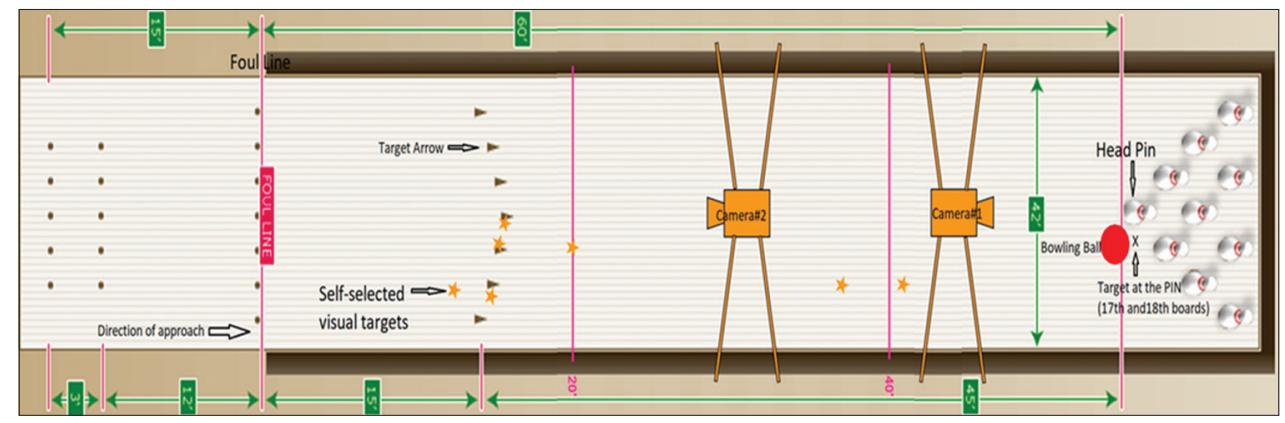

Figure 1. Experimental setup. Stars indicate the visual target locations selected by each participant. Two video cameras were positioned in the middle of lane facing towards the pins (Camera\#1) and the foul line (Camera\#2)

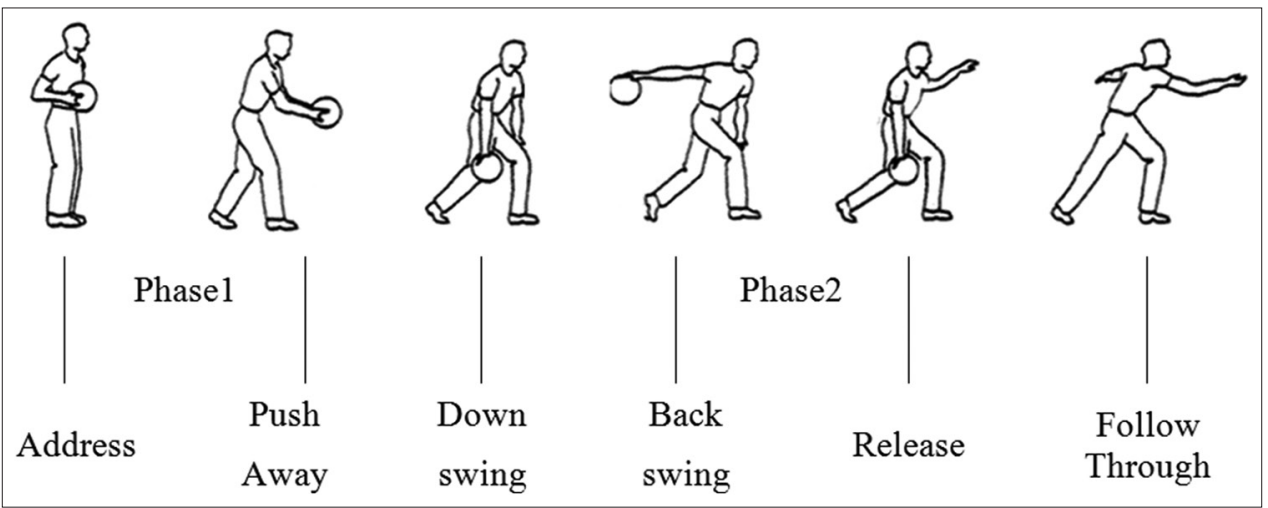

Figure 2. Bowling approach steps and events for eye movement analysis 
compared for accurate and inaccurate trials using a paired t-test (two-tailed). The alpha level for all statistical tests was set at.05.

\section{RESULTS}

\section{Performance}

Most participants preferred to target somewhere near the arrows, located 15 feet down the lane. One participant noted that she focused either 15 or 41 feet down the lane, depending on the oil pattern on the lane. This participant elected to look at 41 feet for the experiment. Absolute and variable error at the visual target and final target were summarized in Table 1.

\section{Visual target error}

In general, visual target error increased as target distance increased (Figure 3). The main effect of visual target distance on absolute error at preliminary visual target was significant $[F(3,18)=4.228, p<.05]$, and a post hoc pairwise comparison showed that absolute error at 20 -foot condition was significantly lower than absolute error at 60 -foot condition $(p<.05)$. The main effect of visual target distance on spatial variability at visual target was statistically significant $[F(3,18)=11.248, \mathrm{p}<.01]$. Post hoc comparisons indicated that variable error was significantly lower at the 20 -foot and self-selected condition as compared to the 40-foot and 60foot condition $(p s<.01)$.

\section{Final target error}

The main effect of visual target distance on absolute error at final target was statistically significant $[F(3,18)=4.079, p<.05]$, however, its effect on variable error did not reach statistical significance $[F(3,18)=3.040, p=.056]$ (Figure 4). A post hoc comparison showed that self-selected condition was more accurate at pins compared to the 40-foot intermediate target condition $(p<.05)$. In detail, absolute and variable error at final target was the least in the participants' preferred target distance condition and second lowest in 60-foot condition, in comparison to the other two intermediate target distance conditions.

\section{Eye Fixations}

Due to device malfunction eye movement data was only available for 6 participants. The duration of the last fixation before the initiation of push-off, the last fixation before ball release, and the average fixation from push-off to ball release were compared (Table 2).

The main effect of visual target distance on fixation duration before initiation of push off was statistically significant $[F(3,18)=5.121, \mathrm{p}<.01]$. Fixation duration before push-away

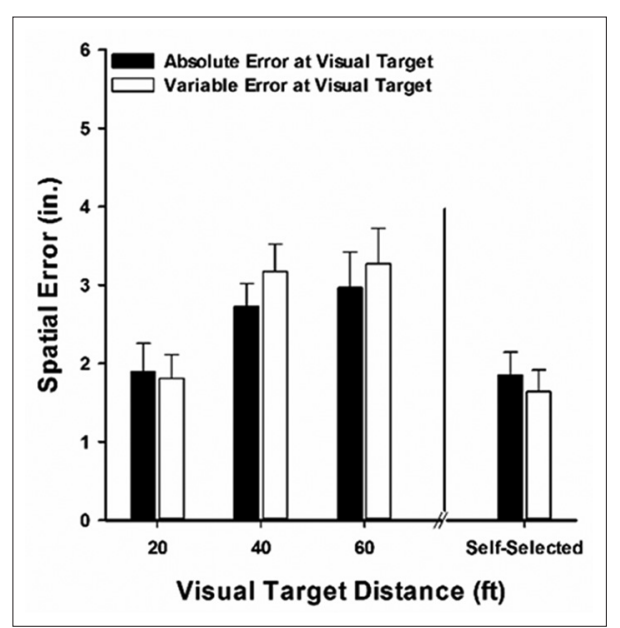

Figure 3. Absolute error and Variable error at visual target.

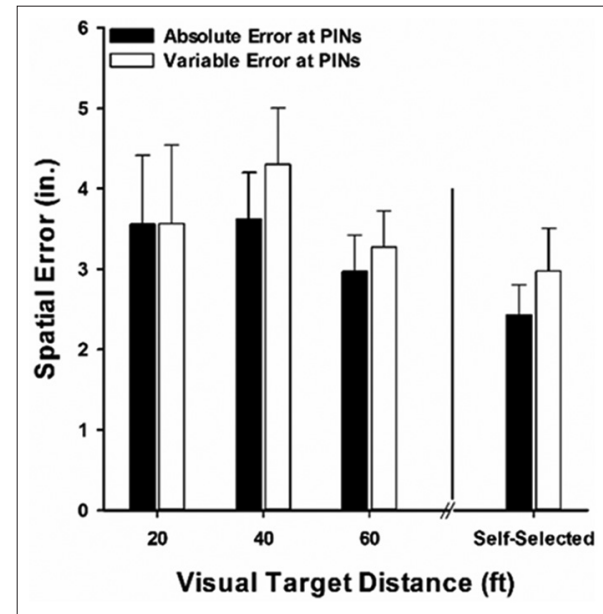

Figure 4. Absolute error and Variable error at PINs.

Table 1. Performance accuracy at visual target and PINs (inch)

\begin{tabular}{|c|c|c|c|c|c|}
\hline \multirow[t]{2}{*}{ Location } & \multirow[t]{2}{*}{ Error } & \multicolumn{4}{|c|}{ Visual target distance (ft) } \\
\hline & & 20 & 40 & 60 & Self- selected \\
\hline \multirow[t]{4}{*}{ Visual target } & $\mathrm{AE}$ & & & & \\
\hline & Mean \pm SD & $1.89 \pm 0.97$ & $2.73 \pm 0.76$ & $2.96 \pm 1.19$ & $1.85 \pm 0.77$ \\
\hline & VE & & & & \\
\hline & Mean \pm SD & $1.80 \pm 0.80$ & $3.17 \pm 0.92$ & $3.27 \pm 1.18$ & $1.64 \pm 0.72$ \\
\hline \multirow[t]{4}{*}{ Target (PINs) } & $\mathrm{AE}$ & & & & \\
\hline & Mean \pm SD & $3.55 \pm 2.27$ & $3.62 \pm 1.53$ & $2.96 \pm 1.19$ & $2.42 \pm 0.99$ \\
\hline & VE & & & & \\
\hline & Mean \pm SD & $3.56 \pm 2.59$ & $4.30 \pm 1.85$ & $3.27 \pm 1.18$ & $2.97 \pm 1.39$ \\
\hline
\end{tabular}

$\mathrm{AE}=\mathrm{Absolute}$ error. $\mathrm{VE}=$ Variable error. 
Table 2. Fixation duration and frequency $(\mathrm{sec})$

\begin{tabular}{|c|c|c|c|c|c|}
\hline & Fixation & & Visual targ & istance $(\mathrm{ft})$ & \\
\hline & & 20 & 40 & 60 & Self -selected \\
\hline Duration & Before movement initiation (push-away) & & & & \\
\hline & Mean \pm SD & $0.325 \pm 0.141$ & $0.339 \pm 0.102$ & $0.413 \pm 0.152$ & $0.299 \pm 0.076$ \\
\hline & Before ball release & & & & \\
\hline & Mean \pm SD & $0.127 \pm 0.021$ & $0.130 \pm 0.020$ & $0.131 \pm 0.021$ & $0.125 \pm 0.026$ \\
\hline & Average & & & & \\
\hline & Mean \pm SD & $0.196 \pm 0.026$ & $0.190 \pm 0.016$ & $0.195 \pm 0.031$ & $0.203 \pm 0.035$ \\
\hline Frequency & Average & & & & \\
\hline & Mean \pm SD & $6.683 \pm 0.907$ & $6.825 \pm 0.446$ & $6.208 \pm 0.890$ & $6.716 \pm 1.305$ \\
\hline
\end{tabular}

was longer at the 60 -foot condition compared to 20 -foot condition $(p<.05)$. However, fixation duration before ball release showed no differences relative to visual target distance conditions. Fixation frequency was not influenced by visual target distance. The final fixation before movement initiation was significantly longer than the average fixation (for example, $p=0.03$ for the 60 -foot condition), which was in turn significantly longer than the last fixation before ball release $(p s<.01)$ (Figure 5). Average fixation duration was longer than fixation duration before ball release in all visual target distance conditions ( $p s<.01)$.

\section{Fixations and accuracy}

Final fixation duration was no longer on accurate trials compared to those of inaccurate trials. Final fixation duration was compared in accurate and inaccurate trials, which were defined as shots either within 1 inch of the preliminary target or within 1 inch of the final target (the $17.5^{\text {th }}$ board 60 feet down the lane). Final fixation duration was almost identical in all cases, as shown in Figure 6.

\section{DISCUSSION}

\section{Spatial Accuracy of Aiming}

The magnitude and variability of ball deviation from the visual point of aim increased as visual aim point was located closer to the pins. Though practice time was limited at each of the visual target distances, participants in this study presented intermediate skill level and were successful in projecting the bowling ball towards the location of aim with minimal variability. Under these assumptions, increases in spatial error at the visual point of aim may be associated with task difficulty, which increases with target distance. While spatial error at the visual target increased with aiming distance, final target error (i.e., deviation from the head pin) was greater at 20 -foot and 40 -foot conditions than 60 -foot condition. Moreover, similarities in final target error between the 60 -foot condition and the self-selected condition have been determined. These findings indicate that placing aim location directly on the pin could reduce the final target error and suggest an alternative strategy compared to aiming at an intermediate space. If more than one visuo-motor task space is available, such as in bowling, directing visual fo-

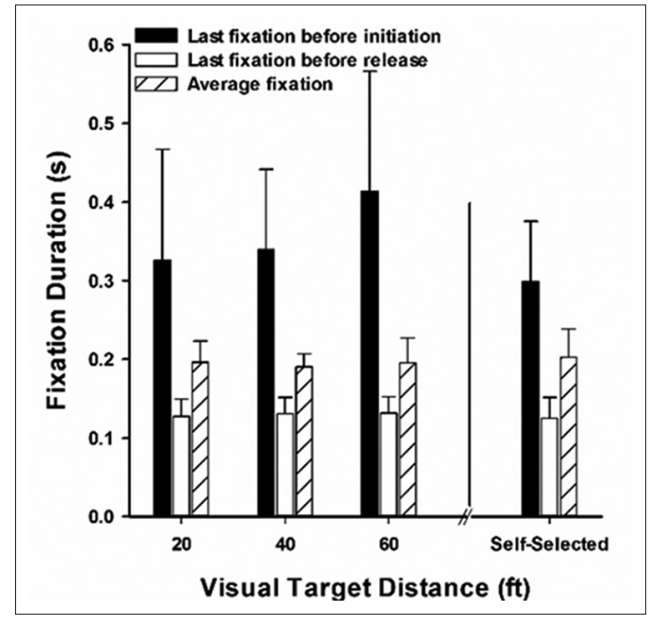

Figure 5. Final fixation duration at two movement events and Average fixation duration.

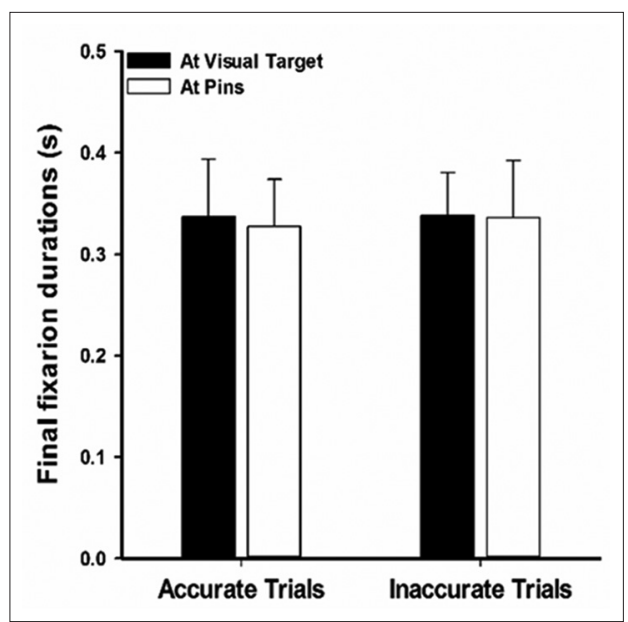

Figure 6. Fixation duration with ball accuracy at visual target and at pins.

cus on the target rather than the intermediate space would be beneficial in terms of accuracy, unless deliberate practice has been provided to aim at a fixed reference spot (e.g., aim spot on the lane). Mackenzie et al. (2011) reported no significant differences in success rate as well as putter speed variability between visually focusing on the hole and looking at the ball in golf putting. Such findings suggest that visual point of aim on the target played a crucial role in focusing the golf- 
er's attention to the outcome of movement execution such as "rolling the ball toward the hole". Given that the variable error at the final target (i.e., pin) was smaller in the 60 -foot condition, additional research is necessary, across all levels of expertise, in order to understand the effect of visual focus training on a far-target aiming task.

\section{Gaze Control Characteristics}

Gaze fixation duration before the initiation of push-off was longer compared to before ball release and average fixation duration, regardless of the distance of visual aim point. A longer visual target distance (i.e., 60-foot condition) caused constrained gaze control, which is indicated by increased fixation duration. In the current study, this increased fixation duration is consistent with previous research on far-target aiming (Behan \& Wilson, 2008; Henry \& Rogers, 1960; Vickers, 1996, 2009; Williams, Singer, \& Frehlich, 2002), where longer fixation duration has been shown to be associated with increased task difficulty and anxiety level. The findings herein agree with Vickers (2007), who demonstrated the importance of acquired visual information during the movement preparation period. However, the task-specific nature of this gaze control characteristic, has been reported, with limitations exposed, particularly when visual occlusion is accompanied or continuous control of movement is required in aiming tasks (De Oliveira, Oudejans, \& Beek, 2006; Glöckner, Heinen, Johnson, \& Raab, 2012; Oudejans, van de Langenberg, \& Hutter, 2002). Gaze fixation duration during the movement preparation period was not different in terms of aiming accuracy (i.e. accurate trials vs. inaccurate trials). This finding is inconsistent with observations seen in studies on other aiming tasks, where a significant relationship between performance accuracy and gaze fixation duration was found (Causer, Bennett, Holmes, Janelle, \& Williams, 2010; Vickers, 1992; Williams et al., 2002; Wilson \& Pearcy, 2009). This disparity between such findings may have resulted from the amount of visual information and number of necessary parameters to be controlled during movement execution, and consequences on performance outcome. Future studies should examine gaze fixation duration-performance accuracy relationship, with the consideration of the performers skill level and the number of visuo-motor task space. An alternative view on gaze fixation (occurs before movement initiation) emphasized its significance as a reflection of the efficient processing and anticipation of object location and object characteristics, as well as the acceleration of transitioning focus of attention facilitating the transition (Horn, Okumura, Alexander, Gardin, \& Sylvester, 2012). This notion is supported through studies showing that the role of a longer gaze fixation during movement preparation could be limited to tasks requiring high demands of information processing and/or re-parameterization of movement, in order to maintain trial-to-trial consistency (Horn et al., 2012; Klostermann, Kredel, \& Hossner, 2013).

This pilot study has several limitations, including limited sample size and differences in preferred visual aiming points between participants. The generalizability of this pilot study is also limited by the skill-based sample of bowlers. Skilled bowlers were recruited in this study to minimize the source of variation originating from throwing motion, and therefore the potential influence on the aiming accuracy. Therefore, different pattern of gaze control may appear, especially, in the people in the lower skill level, who has to allocate a significant amount of attention to the delivery itself (Shank \& Lajoie, 2013). Consequently, future research is needed in order to replicate the present findings with different individual as well as task context.

\section{CONCLUSION}

The aim of the current study was to directly manipulate the distance of visual focus of attention in a far-target aiming task with experienced bowlers. The present study examined gaze fixation duration and aiming accuracy as a function of the distance of visual focus of attention in far-target aiming. Our results failed to demonstrate the effect of task difficulty (e.g., visual target distance) and performance accuracy on the gaze fixation duration in the movement preparation period. However, the results provided further evidence on the critical role of processing visual information before the execution of movement. The preliminary results of this study imply that visual focus on a far-distance target, instead of an intermediate space, could result in an advantageous effect on performance outcome, especially, with the presence of more than one visuo-motor task space. Future studies should seek to elucidate whether this relationship is consistent across various skill levels, as well as context of aiming tasks.

\section{REFERENCES}

Behan, M., \& Wilson, M. (2008). State anxiety and visual attention: The role of the quiet eye period in aiming to a far target. Journal of Sports Sciences, 26(2), 207-215.

Bell, J. J., \& Hardy, J. (2009). Effects of attentional focus on skilled performance in golf. Journal of Applied Sport Psychology, 21(2), 163-177.

Causer, J., Bennett, S. J., Holmes, P. S., Janelle, C. M., \& Williams, A. M. (2010). Quiet eye duration and gun motion in elite shotgun shooting. Medicine \& Science in Sports \& Exercise, 42(8), 1599-1608.

Cockerill, I. M. (1979). Visual control in golf putting. In C. H. Nadeau, W. R. Halliwell, K. M. Newell, \& G. C. Roberts (Eds.), Psychology of motor behavior and sport (pp. 377-384). Champaign, IL: Human Kinetics.

De Oliveira, R. F., Oudejans, R. R., \& Beek, P. J. (2006). Late information pick-up is preferred in basketball jump shooting. Journal of Sports Sciences, 24(9), 933-940.

Glöckner, A., Heinen, T., Johnson, J. G., \& Raab, M. (2012). Network approaches for expert decisions in sports. $\mathrm{Hu}$ man Movement Science, 31(2), 318-333.

Henry, F. M., \& Rogers, D. E. (1960). Increased response latency for complicated movements and a "memory drum" theory of neuromotor reaction. Research Quarterly. American Association for Health, Physical Education and Recreation, 31(3), 448-458.

Horn, R. R., Okumura, M. S., Alexander, M. G., Gardin, F. A., \& Sylvester, C. T. (2012). Quiet eye duration is responsive to variability of practice and to the axis of 
target changes. Research quarterly for exercise and sport, 83(2), 204-211.

Klostermann, A., Kredel, R., \& Hossner, E.-J. (2013). The "quiet eye" and motor performance: Task demands matter! Journal of Experimental Psychology: Human Perception and Performance, 39(5), 1270-1278.

Lohse, K. R., Sherwood, D. E., \& Healy, A. F. (2010). How changing the focus of attention affects performance, kinematics, and electromyography in dart throwing. $\mathrm{Hu}$ man Movement Science, 29(4), 542-555.

Mackenzie, S. J., Foley, S. M., \& Adamczyk, A. P. (2011). Visually focusing on the far versus the near target during the putting stroke. Journal of Sports Sciences, 29(12), 1243-1251.

Marchant, D. C., Clough, P. J., \& Crawshaw, M. (2007). The effects of attentional focusing strategies on novice dart throwing performance and their task experiences. International Journal of Sport and Exercise Psychology, 5(3), 291-303.

McKay, B., \& Wulf, G. (2012). A distal external focus enhances novice dart throwing performance. International Journal of Sport and Exercise Psychology, 10(2), 149-156.

Oudejans, R. R., van de Langenberg, R. W., \& Hutter, R. V. (2002). Aiming at a far target under different viewing conditions: Visual control in basketball jump shooting. Human Movement Science, 21(4), 457-480.

Porter, J. M., Anton, P. M., Wikoff, N. M., \& Ostrowski, J. B. (2013). Instructing skilled athletes to focus their attention externally at greater distances enhances jumping performance. The Journal of Strength \& Conditioning Research, 27(8), 2073-2078.

Razman R., Abas W.A.B.W., Osman N.A.A., Cheong J.P.G. (2011). Temporal Characteristics of the Final Delivery Phase and Its Relation to Tenpin Bowling Performance. In: Osman N.A.A., Abas W.A.B.W., Wahab A.K.A., Ting HN. (eds) $5^{\text {th }}$ Kuala Lumpur International Conference on Biomedical Engineering 2011. IFMBE Proceedings, vol 35. Springer, Berlin, Heidelberg.

Shank, V. \& Lajoie, Y. (2013). Attentional demands in the execution phase of curling in novices and experts. International Journal of Kinesiology \& Sports Science, 1(1), 1-8.

Slowinski, J. (2007, June). 3-Point targeting for advanced lane play. [Online forum post]. Retrieved from https:// www.bowlingthismonth.com/bowling-tips/lane-playalignment

Taylor, D. (1979). Secret of Bowling Strikes: Wilshire Book Company.

Thomas, P. R., Schlinker, P. J., \& Over, R. (1996). Psychological and psychomotor skills associated with prowess at ten-pin bowling. Journal of Sports Sciences, 14(3), 255-268.

Vickers, J. N. (1992). Gaze control in putting. Perception, 21(1), 117-132.

Vickers, J. N. (1996). Visual control when aiming at a far target. Journal of Experimental Psychology: Human Perception and Performance, 22(2), 342-354.

Vickers, J. N. (2007). Perception, cognition, and decision training: The quiet eye in action: Champaign, IL: Human Kinetics.

Vickers, J. N. (2009). Advances in coupling perception and action: the quiet eye as a bidirectional link between gaze, attention, and action. Progress in brain research, 174, 279-288.

Vickers, J. N. (2011). Mind over muscle: The role of gaze control, spatial cognition, and the quiet eye in motor expertise: Springer.

Vine, S. J., Moore, L. J., \& Wilson, M. R. (2011). Quiet eye training facilitates competitive putting performance in elite golfers. Frontiers in psychology, 2(8), 1-9.

Williams, A. M., Singer, R. N., \& Frehlich, S. G. (2002). Quiet eye duration, expertise, and task complexity in near and far aiming tasks. Journal of Motor Behavior, 34(2), 197-207.

Wilson, M. R., \& Pearcy, R. C. (2009). Visuomotor control of straight and breaking golf putts. Perceptual and Motor Skills, 109(2), 555-562.

Wulf, G. (2007). Attentional focus and motor learning: A review of 10 years of research. E-journal Bewegung und Training, 1(2-3), 1-11.

Wulf, G., Dufek, J. S., Lozano, L., \& Pettigrew, C. (2010). Increased jump height and reduced EMG activity with an external focus. Human Movement Science, 29(3), 440-448.

Wulf, G., \& Lewthwaite, R. (2010). Effortless motor learning? An external focus of attention enhances movement effectiveness and efficiency. In B. Bruya (Ed.), Effortless attention: A new perspective in attention and action (p. 75-101). Cambridge, MA: MIT Press.

Wulf, G., McNevin, N., \& Shea, C. H. (2001). The automaticity of complex motor skill learning as a function of attentional focus. The Quarterly Journal of Experimental Psychology: Section A, 54(4), 1143-1154.

Wulf, G., \& Su, J. (2007). An external focus of attention enhances golf shot accuracy in beginners and experts. Research quarterly for exercise and sport, 78(4), 384-389.

Young, K. C., Sherk, V. D., \& Bemben, D. A. (2011). Inter-limb musculoskeletal differences in competitive tenpin bowlers: A preliminary analysis. Journal of Musculoskeletal Neuronal Interact, 11(1), 21-26. 